\title{
Transformación de prácticas de trato postcosecha de la guanábana para fortalecer identidad local y comercialización
}

\section{Transformation of post-harvest treatment practices of the soursop to strengthen local identity and commercialization}

\author{
US Daniel Lopera Molano ${ }^{1}$ \\ Ángela María Lopera Molano² \\ Tatiana Hernández Cifuentes ${ }^{3}$ \\ Yorladys Mayilsen Martínez Aroca ${ }^{4}$ \\ Carlos Mauricio Santana Sáenz ${ }^{5}$ \\ Marly Viviana Tafur Osorio ${ }^{6}$
}

(e) Recepción: $26 / 09 / 2019$

( Aprobación: 18/10/2019

C Publicación: 19/12/2019

Para citar este artículo:

Lopera Molano, D., Lopera Molano, A. M., Hernández Cifuentes, T., Martínez Aroca, Y., M., Santana Sáenz, C. M., \& Tafur Osorio, M. V. (2019).

Transformación de prácticas de trato postcosecha de la guanábana para fortalecer identidad local y comercialización. Indagare, (7), 28-35. https://doi.org/10.35707/indagare/704

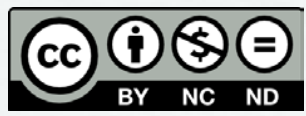

${ }^{1}$ Grupo MYSCO. Universidad de Ibagué. ORCID: 0000-0002-0793-8478. daniel.lopera@unibague.edu.co

${ }^{2}$ Grupo MYSCO. Universidad de Ibagué. ORCID: 0000-0002-3797-7996. angela.lopera@unibague.edu.co

${ }^{3}$ Grupo UNIDERE. Universidad de Ibagué. ORCID: 0000-0002-9992-3176. tatiana.hernandez@unibague.edu.co

${ }^{4}$ Grupo NATURATU. Universidad de Ibagué. ORCID: 0000-0002-3581-988X. yorladys.martinez@unibague.edu.co

${ }^{5}$ Grupo UNIDERE. Universidad de Ibagué. ORCID: 0000-0003-3230-0211. carlos.santana@unibague.edu.co

${ }^{6}$ Grupo MYSCO. Universidad de Ibagué. ORCID: 0000-0002-5580-6410. marly.tafur@unibague.edu.co 


\title{
Resumen
}

En este artículo se presenta un avance de los resultados obtenidos del proyecto de desarrollo tecnológico titulado "Transformación de las prácticas de trato postcosecha de la guanábana para fortalecer identidad local y comercialización", en el desarrollo de tres de sus cuatro fases. Los enfoques que han orientado el proceso son el diseño participativo y el pensamiento sistémico. El proyecto se ha desarrollado junto con la Asociación Aprofruverf del municipio de Fresno, en donde se desplegaron distintos dispositivos transformadores de prácticas, que han fortalecido los procesos asociativos e identitarios de los agricultores, y que han redundado en el mejoramiento de su calidad de vida a través de propuestas concretas para el mejoramiento de la postcosecha y la comercialización.

\begin{abstract}
This article presents a preview of the outcomes obtained from the technological development project entitled "Transformation of post-harvest treatment practices of the Soursop to strengthen local identity and commercialization", in the development of three of its four phases. The approaches that have guided the process are participatory design and systemic thinking. The project has been developed together with the Aprofruverf Association of the municipality of Fresno, Colombia, where different transforming practice devices were deployed, which have strengthened the associative and identity processes of the farmers, and which have resulted in the improvement of their quality of life through concrete proposals for postharvest improvement and commercialization.
\end{abstract}

Palabras claves: Diseño, guanábana, comercialización, identidad.

Key words: Design, soursop, commercialization, identity.

\section{Introducción}

Este proyecto muestra la importancia de la articulación intrainstitucional para el desarrollo de procesos de investigación, docencia y extensión, que fortalecen la relación universidad-sociedad, en aras de contribuir al desarrollo regional; esto a través de una experiencia situada en el contexto rural, que favoreció la articulación y participación de la oficina de Responsabilidad Social Integral, estudiantes y asesores del Semestre Paz y Región, vinculación de estudiantes tesistas del programa de Diseño, Comunicación Social y Periodismo y la participación de tres grupos de investigación interdisciplinarios de la Universidad. 
Al considerar los problemas centrales que afectan a los pequeños productores asociados a Aprofruverf en el municipio de Fresno, tales como las precarias condiciones de infraestructura que afectan el buen trato de la fruta dentro de los cultivos (vastas zonas con pendientes muy inclinadas); ausencia de políticas y acciones que regulen los precios de los productos agrícolas en el mercado; falta de apoyo del gobierno para menguar los picos bajos de producción; pérdidas de cosecha y bajos precios de sus productos en el mercado; entre otros factores que afectan la comercialización, se planteó como pregunta central: ¿cómo transformar procesos de trato postcosecha y distribución de la guanábana para la valorización del producto, integrando estrategias de identidad territorial?

El proyecto se abordó desde tres campos de investigación relacionados. El primero es el Desarrollo Territorial Rural (DTR), entendido como un proceso local, endógeno y descentralizado "[...] de transformación productiva e institucional de un espacio rural determinado, cuyo fin es reducir la pobreza rural” (Schejtman \& Berdegué, 2004, p. 30). El segundo es la transformación de prácticas y diseño participativo (IDEO, 2015), entendidas como la transición de un sistema complejo de actividades, que parte de las situaciones que se desean cambiar, hacia algo considerado como mejor, a partir del reconocimiento de propósitos compartidos y generación de estándares y criterios reconocidos por las comunidades involucradas y consideradas valiosas para el desarrollo y creación constante de nuevas y mejoradas condiciones. El tercero es la Identidad Cultural Territorial (Uribe, 2006), entendida como un sistema relacional entre vínculos de cohesión, desde la identidad y la memoria de los territorios, y sus propios sistemas simbólicos de producción. Los tres campos se relacionan con la intención de comprender las prácticas actuales que se realizan y se transforman colectivamente para la valorización, por parte de los actores implicados y no solo del consumidor final, de un producto que se cultiva en esa región, como la guanábana.

De acuerdo con lo anterior, el diseño participativo de dispositivos transformadores de prácticas (Campos, 1999), sistema articulado de piezas comunicacionales, empaques, estrategias de integración asociativa y de redes institucionales, entre otros, se despliega como estrategia para el fortalecimiento de la identidad local y la territorialización del desarrollo, que de manera integrada, redunda en el mejoramiento de las condiciones de vida, ligadas al fortalecimiento de la economía campesina en los proceso de postcosecha y comercialización.

\section{Materiales y métodos}

El proyecto, en su etapa inicial, partió de la construcción de un contexto de familiarización, a partir de visitas de campo y entrevistas a actores involucrados, que favoreció una primera 
Para responder a esta situación, desde el proyecto y su visión de Desarrollo Territorial Rural, esta experiencia se ha trazado varios retos: creación y fortalecimiento de una red de trabajo con la comunidad y entidades territoriales, academia, ANDI (Asociación Nacional de Empresarios de Colombia), Asohofrucol (Asociación Hortofrutícola de Colombia), Sena, etc., que permitan a la comunidad coconstruir propuestas de desarrollo local con una agenda de trabajo que responda a sus necesidades. De otro lado y para fortalecer procesos asociativos, se han diseñado dispositivos de diálogo y toma de decisiones, cartillas de memoria del proceso, fortalecimiento de las Escuelas de Campo, diseño de piezas comunicativas para fortalecer la identidad local.

De la mano de lo anterior, el equipo investigador también está articulando estos múltiples esfuerzos para apoyar en la materialización de infraestructuras para el transporte de la guanábana dentro de los cultivos (garruchas) y experiencias de comercialización con supermercados de la ciudad de Ibagué, lo que desembocó en el diseño y estructuración de un proyecto para la ADR (Agencia de Desarrollo Rural), enfocado a "Implementar sistemas de infraestructura y comercialización para mejorar las condiciones del proceso productivo de guanábana a los productores campesinos asociados a Aprofruverf (Asociación de Productores de Frutas y Verduras de Fresno)". Para este último dispositivo, se ha trabajado en un fuerte proceso de articulación con dependencias de la Universidad de Ibagué.

\section{Resultados}

Los diseños de dispositivos transformadores de prácticas que se han desarrollado en las tres primeras fases del proyecto son: 1) creación y fortalecimiento de red institucional para el apoyo y acompañamiento de los proyectos comunes propuestos por la comunidad campesina asociada en Aprofruverf (de donde emerge el presente proyecto); 2) coconstrucción de las Escuelas de Campo como escenarios para la convergencia de la comunidad y de las distintas instituciones, para la toma de decisiones y diálogo, así como el apoyo en asistencia técnica integral en los cultivos y acompañamiento socio empresarial; 3) fortalecimiento de la memoria del proceso a través del desarrollo de unas cartillas construidas en cada una de las Escuelas de Campo realizadas durante el 2019;4) codiseño del logo de la asociación como favorecedor del reconocimiento de la identidad de Aprofruverf, que a su vez articula y fortalece procesos futuros de comercialización, diseño de tarjetas de presentación, gorras, camisetas, etc.; 5) desarrollo colectivo de la propuesta de valor; 6) desarrollo de piezas comunicativas para construcción dela propuesta de valor centrada en historias de vida de los campesinos asociados en Aprofruverf; 7) estructuración de proyecto (entendido como un dispositivo transformador de prácticas) 
para presentación a la ADR, enfocado a implementar sistemas de infraestructura y comercialización para mejorar las condiciones del proceso productivo de guanábana en la asociación Aprofruverf; y 8) diseño y comprobaciones de un empaque para la guanábana que cumpla labores de contención, protección e información.

Los dispositivos desarrollados presentan claros indicios de transformar prácticas para la valorización del producto. De lo anterior se extraen algunas conclusiones, que se presentan a continuación para la comprensión más amplia de los resultados:

- Tres desarrollos articulados se evidencian como necesarios para avanzar en ellos conjuntamente: asociatividad, comercialización e infraestructura; en tres principios relacionales: económicamente justo, ambientalmente responsable y culturalmente viable.

- Los desarrollos específicos en asociatividad e identidad local se han constituido como la estrategia central de aporte a la transformación de prácticas de postcosecha y distribución.

- La construcción y desarrollo del proyecto en red ha permitido ir cumpliendo estos principios relacionales con el aporte de otras disciplinas y entidades.

- La valorización de la asociación, y no solo del producto, ha sido significativa para la transformación colectiva como se muestra en la transformación de las Escuelas de Campo, cuando la didáctica deja de estar centrada en el producto y pasa a estar centrada en la asociación como un todo.

Figura 2. Miembros de la asociación construyendo cartografía social durante una Escuela de Campo
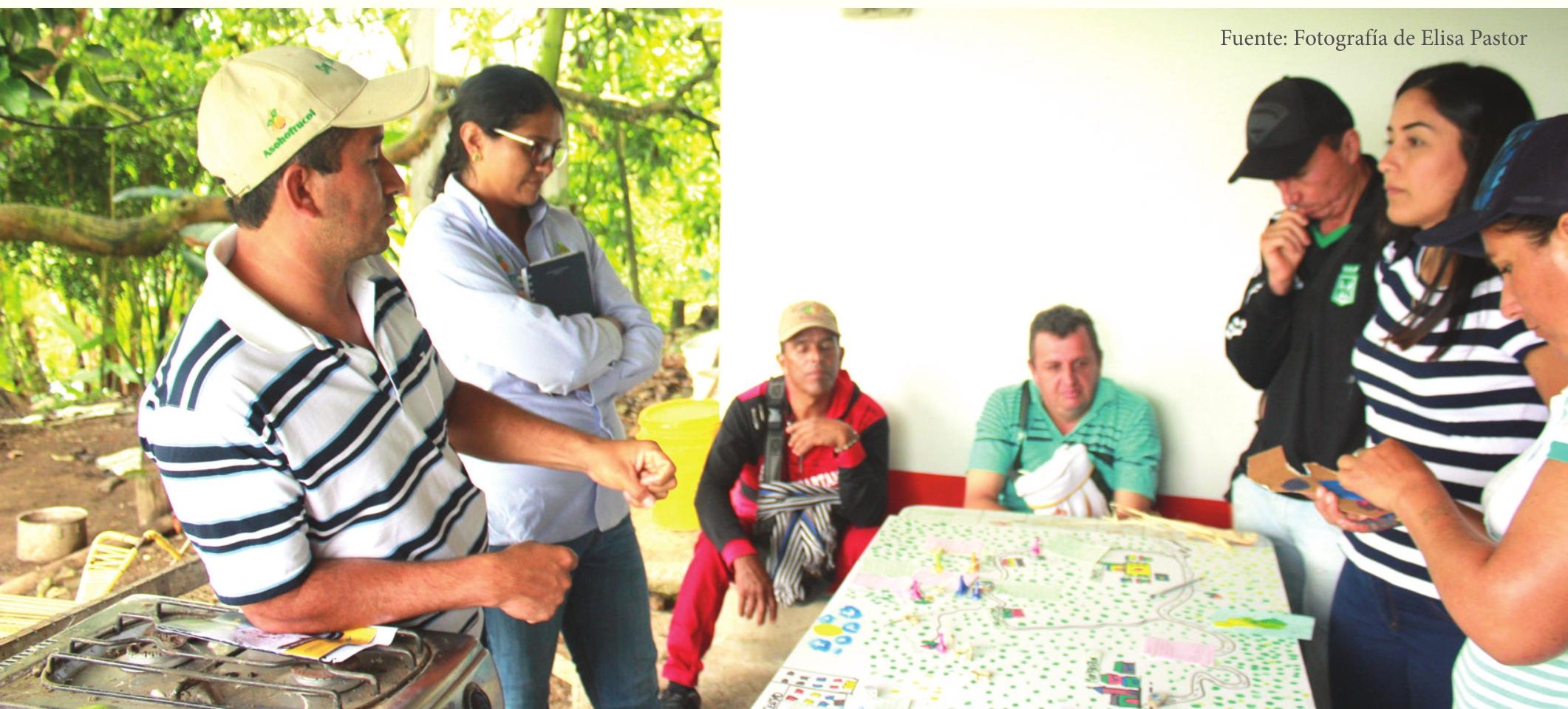


\section{Potencial uso}

Este proyecto desarrolla una apuesta metodológica novedosa en relación a la construcción de trabajo en red con organizaciones comunitarias, académicas, políticas, estatales, pero que además tributan al conocimiento relacionado con el fortalecimiento de la relación universidad-comunidad rural, a partir de la configuración de un sistema de acciones que, vistos de manera sistémica, apuntan al desarrollo de autonomía de las comunidades, por un lado, y al cumplimiento de la responsabilidad social de la academia, por el otro.

La apuesta metodológica del proyecto es una contribución al trabajo interdisciplinar, en tanto se articularon saberes y conocimientos propios del diseño, la comunicación, administración y las ciencias sociales. Esto favoreció una mirada sistémica, que abre caminos para desfragmentar la lectura de la realidad, en tanto que los problemas sociales en su complejidad no tienen como tal una identidad disciplinar, y por eso la manera de abordarlos desde distintas miradas lo hace más enriquecedor.

Concretamente, se aporta con diseño industrial novedoso para favorecer la comercialización de la fruta, memorias de proceso ancladas a la formación y al tejido comunitario, construcción de redes de trabajo para apalancar proyectos y desarrollo de imagen desde ejercicios participativos. Todos estos aportes han sido significativos para la asociación de campesinos del norte del Tolima, y son un referente de apoyo articulado que parte desde las necesidades de los mismos actores para la construcción integral de región.

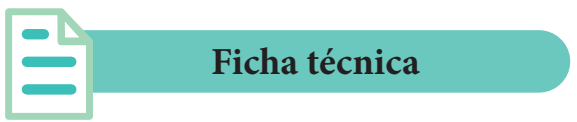

Título del proyecto: Transformación de las prácticas de trato postcosecha de la guanábana para fortalecer identidad local y comercialización.

PRIT: Agroindustria innovadora de base tecnológica.

Código del proyecto: $18-570-\mathrm{INT}$.

Grupo de investigación: MYSCO.

Investigador principal: Daniel Lopera Molano.

\section{Referencias}

Aldana, E., \& Reyes, A. (2006). Disolver problemas. Bogotá, Colombia: Universidad de los Andes.

Campos, G. W. D. S. (1999). Equipes de referência e apoio especializado matricial: um ensaio sobre a reorganização do trabalho em saúde. Ciência \& Saúde Coletiva, 4(2), 393-403. http://dx.doi. org/10.1590/S1413-81231999000200013 
IDEO. (2015). The Field Guide to Human Centered Design, Design Kit. IDEO.org. Recuperado de https://bestgraz.org/wp-content/uploads/2015/09/Field-Guide-to-Human-Centered-Design_ IDEOorg.pdf

Schejtman, A., \& Berdegué, J. (2004). Desarrollo territorial rural. Debates y temas rurales, (1), 7-46. Recuperado de https://www.rimisp.org/wp-content/files_mf/1363093392schejtman_y_ berdegue2004_desarrollo_territorial_rural_5_rimisp_CArdumen.pdf

Uribe, S. (2006). La identidad cultural y el desarrollo territorial rural, una aproximación desde Colombia. Recuperado de http://recursos.salonesvirtuales.com/assets/bloques/Soto_URIBE_ desarrolloterritorialrural.pdf

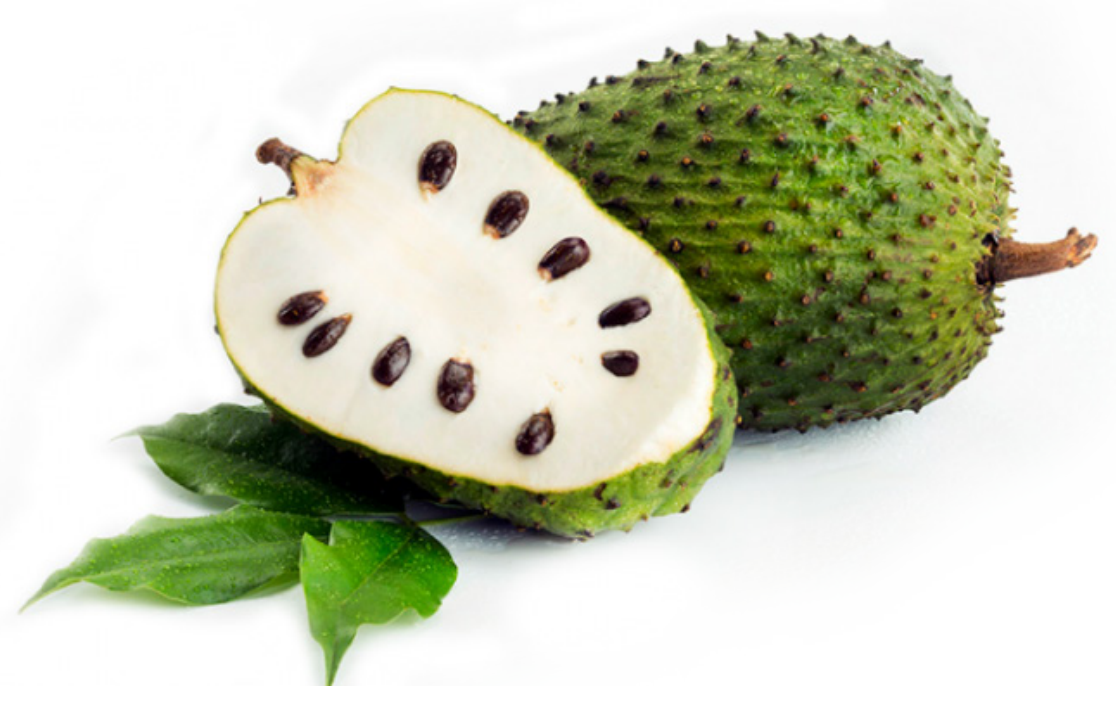

